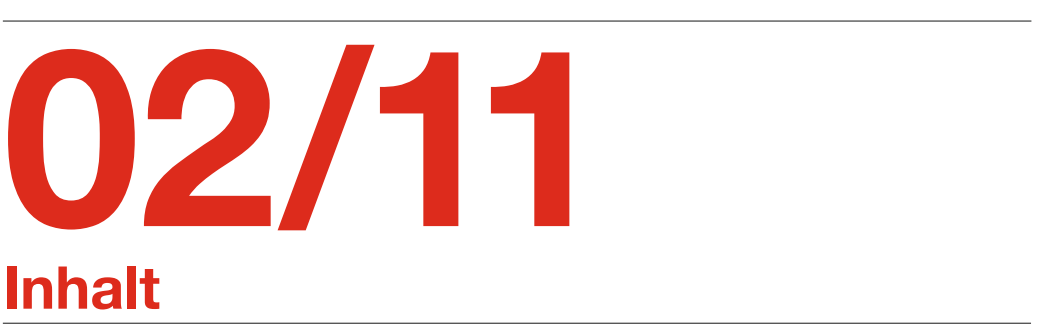

interview

\section{Gesunder Schlaf}

Gespräch mit Univ.-Prof. Dr. Jürgen Zulley, Präsident der Deutschen Akademie für Gesundheit und Schlaf

\section{pharmakotherapie}

10 Qualitätssicherung in der kinder- und jugendpsychiatrischen Psychopharmakotherapie - Einsatz von Therapeutic Drug Monitoring (TDM)

Univ.-Prof. Dr. Andreas Karwautz, cand. med. Stefan Bangratz, Dr. Julia Huemer; Universitätsklinik für Kinder- und Jugendpsychiatrie der Medizinischen Universität Wien

\section{ausbildung}

\section{Europäische Konzepte zur Ausbildung in pädiatrischen} Subspezialitäten - am Beispiel der pädiatrischen Pneumologie, Teil 2

Univ.-Prof. Dr. Maximilian S. Zach, Klinische Abteilung für Pädiatrische Pulmonologie und Allergologie, Universitätsklinik für Kinder- und Jugendheilkunde, Medizinische Universität Graz

\section{osteopathie}

19 Die osteopathische Behandlung des Kindes nach der Geburt

Eva Möckel D.O., Hamburg (Deutschland)

\section{pädagogik}

\section{Das Fehlerkillerprojekt: Leistungsmotivation und} Lernerfolg bei Volksschulkindern

Ergebnisbericht einer Langzeitstudie

Dr. Brigitte Sindelar, Mag. pth. Dorit Hejze, Mag. pth. Vivien Langer; alle: Sigmund Freud PrivatUniversität Wien Paris und Praxis Dr. Sindelar

\section{kinderschutz}

\section{Lost in Translation? - Kooperation im Kinderschutz}

Dr. Alja Goessler ${ }^{1}$, DSA Karlheinz Bonfert ${ }^{2}$, Prim. Univ.-Prof. Dr. Günther Fasching ${ }^{1}$ Abteilung für Kinder- und Jugendchirurgie, Klinikum Klagenfurt

${ }^{2}$ Sozialarbeiter des Eltern- Kindzentrums, Klinikum Klagenfurt

\section{interview}

\section{Babyspeck-Alarm}

Gespräch mit Univ.-Prof. Dr. Karl Zwiauer, Abteilung für Kinder- und Jugendheilkunde, Landesklinikum St. Pölten

\section{Panorama \\ 34 Aktuell \\ 44 Produkte \\ 12 Impressum}

\section{Jahre Kinderklinik}

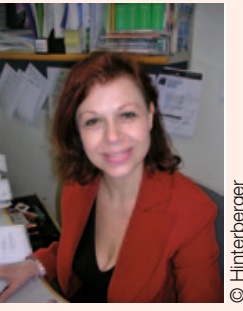

Die Universitätsklinik für Kinder- und Jugendheilkunde Wien feiert inr 100jähriges Bestehen: Aus diesem Anlass findet am 13. Mai in der Hofburg ein Festsymposium statt.

Bekannte Personen aus Wissenschaft und Politik melden sich mit Statements und Grußworten zu Wort, in Festvorträgen gehen Experten auf den historischen Hintergrund und die Entwicklung der Wiener Kinderklinik in den vergangenen 100 Jahren ein. Der Bogen spannt sich von der Gründung der Klinik bis zur Entstehung des fächerübergreifenden pädiatrischen Zentrums am AKH. Die Festrede widmet sich dem Thema „Die Rolle der Pädiatrie als Motor biomedizinischer Forschung".

100 Jahre Kinderklinik - 45 Jahre Neugeborenenscreening: Im Anschluss an das Festsymposium findet der internationale Kongress „Prevention of Congenital Diseases. Screening Newborns: Current State and Future Challenges" statt.

Last, but not least wird am 14. und 15 . Mai ein Post-Kongress-Symposium zur Geschichte der Kinderheilkunde am Wiener Institut für Geschichte der Medizin abgehalten.

Die Geschichte der Wiener Kinderheilkunde ist untrennbar mit der Universitäts-

Kinderklinik am Allgemeinen Krankenhaus - kurz AKH - verbunden.

Auch Pädiatrie \& Pädologie hat eine lange Geschichte und ist untrennbar mit der österreichischen Pädiatrie verbunden. Sie halten Band 46/ Heft 2 in Händen.

Wir, das Team von „Pädiatrie \& Pädologie“, reihen uns unter den Gratulanten ein: Herzlichen Glückwunsch, geschätzte Wiener Kinderklinik!

Prim. Univ.-Prof. Dr. Reinhold Kerbl Dr. Renate Höhl 